\title{
The Structural and Biomechanical Properties of Insect Thick Filaments Expressing Flightin and Cardiac Myosin Binding Protein-C
}

\author{
Lynda Menard $^{1}$, Lori Nyland ${ }^{2}$, Jim Vigoreaux ${ }^{1}$ \\ Department of Biology ${ }^{1}$ and Molecular Physiology and Biophysics ${ }^{2}$, University of Vermont, \\ Burlington, Vermont
}

Cardiac myosin binding protein-c (cMyBP-C) of mammalian cardiac muscle and flightin (FLN) of invertebrate indirect flight muscle (IFM) have been shown to contribute to thick filament stiffness, as determined by calculations of persistence length (PL), an index of flexural rigidity $[1,2]$ in their corresponding muscle systems. FLN and cMyBP-C in vitro bind to a common site in the coiled-coil region of myosin II, and both proteins are known to be regulated by phosphorylation $[3,4]$. To test the hypothesis that FLN and cMyBP-C are functionally homologous, we have determined the extent to which cMyBP-C can rescue the phenotypes manifested in the Drosophila FLN knockout strain $f l^{0}$. Structural characteristics of flight muscle sarcomeres were analyzed by transmission electron microscopy (TEM) and the contour and endto-end length of isolated, hydrated native thick filaments was measured by atomic force microscopy (AFM).

Experiments were carried out on four D. melanogaster mutant and transgenic strains: (i) FLN knockout strain $\left(f l n^{0}\right)$, (ii) a knockout rescued transgenic strain $\left(f l n^{0} ; f n^{+}\right)$, (iii) a transgenic cMyBP-C strain without FLN expression $\left(f \ln ^{0} ; c M y B P C^{+}\right)$, and (iv) a transgenic strain with FLN expression alongside cMyBP-C expression $\left(f \mathrm{fn}^{+} ; \mathrm{cMyBPC} \mathrm{C}^{+}\right)$. In preparation for TEM, thoraces from newly eclosed $(<1$ hour) $D$. melanogaster were bisected, fixed, dehydrated, infiltrated, embedded, sectioned and imaged by TEM [5]. The length of sarcomeres from 4-5 flies for each Drosophila strain was measured using ImageJ. AFM data of isolated thick filaments were evaluated using the parameters and programs described by [6]. Statistical analysis was done using JMP 9 software.

The TEM results confirmed both the sarcomere length measurements and level of structural order previously seen for $f \mathrm{fn}^{0}$ and $f \mathrm{fn}^{0} ; \mathrm{fln}^{+}$, while revealing shorter sarcomeres in the transgenic lines involving cMyBP-C alone (Fig. 1, Table. 1). When cMyBP-C is expressed alongside FLN, sarcomere length is slightly but significantly longer than sarcomere length in the control $f \mathrm{fn}^{0} ; \mathrm{fln}^{+}$. These results support the idea of cMyBP-C binding to myosin in thick filaments of $D$. melanogaster and influencing the length of the filaments. However, the length regulation exerted by cMyBP-C is surpassed by FLN when FLN is present, either by direct binding competition to a common myosin binding site or another regulatory mechanism.

The PL for $f l n^{+} ; c M y B P C^{+}$obtained by AFM was significantly higher than PL for $f l n^{0} ; f n^{+}$ $(2.56 \mu \mathrm{m}$ compared to $1.67 \mu \mathrm{m} ; \mathrm{p}<0.05)$, suggesting that cMyBP-C contributes to filament stiffness when expressed ectopically in IFM. However, the cMyBP-C effect is seen only in the presence of FLN as PL of $f l n^{0} ; c M y B P C^{+}$was not different that PL of $f l n^{0}$. Our observations suggest that the presence of FLN influences the effects that $\mathrm{cMyBP}-\mathrm{C}$ has on the mechanical properties of the thick filaments. This may possibly be due to FLN stabilizing the thick filaments to permit a more ideal environment for cMyBP-C binding. From these studies we conclude that 
ectopically expressed cMyBP-C influence sarcomere length and thick filament stiffness in the flight muscle, consistent with the hypothesis that cMyBP-C and FLN have convergent functions.

The mechanical AFM data complemented the structural TEM data in that it promoted the possibility that competition between the two proteins was likely occurring in the $f l^{+} ; c M y B P C^{+}$ line. While FLN and cMyBP-C have been shown to bind to a common myosin site in vitro, a perspective supported by these AFM/EM observations, cMyBP-C is a very different protein compared to FLN in sequence and size (130 kDa vs. 20kDa) [1, 7]. Our results demonstrate that cMyBP-C may be adjusting structural and mechanical characteristics of myosin thick filaments in the same way as FLN, but not to the same degree.

Our data supports the hypothesis of cMyBP-C being a vertebrate functional homolog to invertebrate FLN. This brings us closer to understanding the role of myosin binding proteins in dictating the structural and mechanical properties of thick filaments, an important determinant of muscle functional properties. Further insight can be gained by elucidation of the molecular interaction between these proteins and the myosin coiled coil.

[1] Ayer, G. and J. O. Vigoreaux (2003). Cell Biochem Biophys 38(1): 41-54.

[2] Nyland, L. R., et al. (2009). Biophys J 96(8): 3273-3280.

[3] Vigoreaux, J. O. (1994). Biochem Genet 32(7): 301-314.

[4] Coulton, A. T. and J. E. Stelzer (2012). Biochem 51(15): 3292-3301.

[5] Barton, B., et al. (2005). J Exp Biol 208(3): 549-560.

[6] Kreplak, L., et al. (2009). J Mol Biol 386(5): 1403-1410.

[7] Miyamoto, C. A., et al. (1999). J Muscle Res Cell Motil 20(7): 703-715

[8] The authors acknowledge the funding of NSF grant MCB 1050834 to J.Vigoreaux.

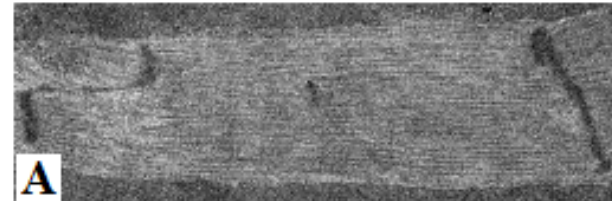

Table 1: Sarcomere and Persistence Length Measurements

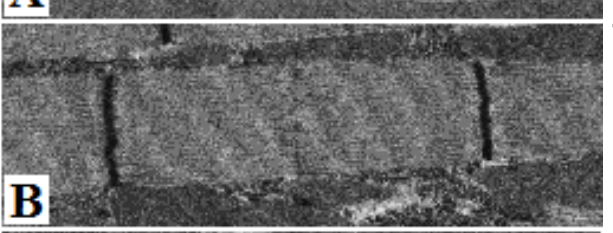

\begin{tabular}{|c|c|c|}
\hline $\begin{array}{c}\text { Transgenic } \\
\text { Strain } \\
\end{array}$ & $\begin{array}{c}\text { Mean Sarcomere } \\
\text { Length }(\mu \mathrm{M}) \pm \mathrm{SEM} \\
\end{array}$ & $\begin{array}{c}\text { Mean Persistence } \\
\text { Length }(\mu \mathrm{M}) \pm \mathrm{SEM} \\
\end{array}$ \\
\hline$f l n^{0}$ & $\begin{array}{c}3.65 \pm 0.03 \\
(\mathrm{n}=141)\end{array}$ & $\begin{array}{c}0.98 \pm 0.14^{\wedge} \\
(\mathrm{n}=105)\end{array}$ \\
\hline$f l n^{0} ; f l n^{+}$ & $\begin{array}{c}3.17 \pm 0.01 * \\
(n=436)\end{array}$ & $\begin{array}{c}1.67 \pm 0.27 \# \\
(\mathrm{n}=26)\end{array}$ \\
\hline$f l n^{0} ; c M y B P C^{+}$ & $\begin{array}{l}2.34 \pm 0.02 * \\
\quad(n=545)\end{array}$ & $\begin{array}{l}0.85 \pm 0.21^{\wedge} \\
\quad(n=44)\end{array}$ \\
\hline $\mathrm{fln}^{+} ; \mathrm{cMyBPC^{+ }}$ & $\begin{array}{c}3.30 \pm 0.01 * \\
(\mathrm{n}=985)\end{array}$ & $\begin{array}{c}2.56 \pm 0.22 * \\
(\mathrm{n}=40)\end{array}$ \\
\hline
\end{tabular}

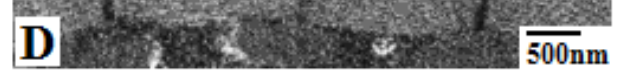

Fig 1. TEM displaying sarcomere morphologies of lines: $f l n^{0}(\mathrm{~A}), f l n^{0} ; f n^{+}(\mathrm{B}), f l n^{0} ; M y B P C^{+}(\mathrm{C})$, and $\mathrm{fln}^{+} ; \mathrm{MyBPC}^{+}$(D). 2500x magnification at $60 \mathrm{kV}$. Scale bar is at $500 \mathrm{~nm}$. 